\title{
Osteopontin's colocalization with the adhesion molecule CEACAM5 in cytoplasm of carcinoma of tongue and its correlation with the invasion of that diease
}

\author{
Fan Zhang ${ }^{1}$, Xu Jin Liu' ${ }^{2}$ Xun $\mathrm{Qu}^{3}$, Zhen Sheng Hu${ }^{1}$, Yong Mei Yang ${ }^{4}$, Ling Ma ${ }^{1}$, Pei Liu' ${ }^{1}$ Ping Shi ${ }^{1}$ \\ and Feng Cai Wei ${ }^{5^{*}}$
}

\begin{abstract}
The purpose of this study was to investigate the expression of carcinoembryonic antigen-related cell adhesion molecule 5 (CEACAM5) and correlate it with OPN expression and function in squamous carcinoma of tongue. Paraffin were sections of 80 samples with squamous carcinoma of tongue and 40 samples with normal tissue of tongue for benign lesion having undergone surgery. Immunohistochemistry (IHC) was used to study the distribution of CEACAM5 and OPN, and double-labeling immunohistochemistry was used to observe the relationship between CEACAM5 and OPN expression.

CEACAM5 and OPN are found in normal tissue of tongue, but with different expression pattern. CEACAM5 expression mainly with membranous staining is restricted on the superficial epithelium. However, OPN expression with mainly cytoplasmic staining is restricted on the deep epithelium. No colocalization of CEACAM5 and OPN have been observed in normal tissue of tongue. In squamous carcinoma of tongue, CEACAM5 expression with cytoplasmic staining is different from normal tongue tissue with membranous staining, and the transformation of CEACAM5 distribution from membrane to cytoplasm is an important incident for the invasion and differentiation of tumor. CEACAM5 and OPN are colocalized in cytoplasm, and a significant correlation was observed between the positive colocalization and the negative colocalization in the depth of invasion and the differentiation of the tumor.
\end{abstract}

Keywords: CEACAM5, OPN, Carcinoma of tongue, Colocalization

\section{Introduction}

Homeostasis in normal tissue is regulated by a balance between proliferative activity and cell loss by apoptosis $[1,2]$. Attachment to correct extracellular matrix (ECM) is essential for survival and growth of normal adhering cells, whereas cancer cells are able to abrogate this requirement. Several growth factors and cytokines play pivotal roles in the regulation of growth and survival of neoplastic cells through affecting integrin-mediated adhesion to ECM.

Cell adhesion molecules are important mediators of cellular contacts and cellular polarity that also modulate

\footnotetext{
*Correspondence: weifengcai2012@126.com

${ }^{5}$ Oral and Maxillofacial Surgery of Qilu hospital of Shandong University, 107\#, Wenhua Xi Road, Jinan, Shandong 250012, People's Republic of China Full list of author information is available at the end of the article
}

proliferation, differentiation, and invasion. Osteopontin (OPN) is a $70-\mathrm{kDa}$ secreted extracellular matrix glycoprotein with an arginine-glycine aspartate-binding motif capable of interaction with integrin subunits [3-5]. Both OPN and CEACAM5 are important cell adhesion molecules. OPN has been demonstrated to be expressed in a variety of human tissues, including the kidneys, thyroid, gastrointestinal tract, breast, and endometrium, and has been implicated in mediation of cell-cell and cellextracellular matrix communication that encompass both normal and tumorigenic developmental processes, cell adhesion, spreading, metastasis, and invasion [6-8]. CEACAM5 is a member of the carcinoembryonic antigen and the immunoglobulin superfamily. The CEACAM5 gene, also known as CD66e, codes for the protein, CEA [9]. CEACAM5 was first described in 1965 
as a gastrointestinal oncofetal antigen [10], but is now known to be overexpressed in a majority of carcinomas, including those of the gastrointestinal tract, the respiratory and genitourinary systems, and breast cancer [11-15]. Studies have demonstrated that OPN is colocalized with the CEACAM1 and enhances invasion of CEACAM1 expressing cells [16]. At present, no information is available on the expression of CEACAM5 and OPN in squamous carcinoma of tongue. Our study would at evaluating the colocalization of OPN with CEACAM5 and the correlation with the carcinoma of tongue invasion and the degree of differentiation.

\section{Materials and methods \\ Patients}

The study included 80 patients with squamous carcinoma of tongue and 40 patients with normal tissue of tongue for benign lesion having undergone primary surgical resection at Qilu Hospital of Shandong University between 2008 and 2010. The clinicopathologic information,including sex, age, tumor stage, and tumor differentiation, was obtained from the clinical records. All the diagnoses were made by three pathologists following the WHO classification of tumors: Pathology and Genetics tumors of digestive system.

\section{Immunohistochemistry(IHC)}

Immunohistochemistry was performed on 4-um-thick routinely processed paraffin sections. CEACAM5 was detected using a mouse monoclonal anti-CEACAM5 antibody (abcam). Sections were dewaxed, and endogenous peroxidase was blocked by immersing the slides in a $3 \%$ solution of hydrogen peroxide in methanol for $10 \mathrm{~min}$, followed by antigen retrieval. The slides were immersed in $0.01 \mathrm{~mol} / \mathrm{L}$ citrate buffer solution $(\mathrm{pH} 6.0)$ and placed in a microwave oven for $25 \mathrm{~min}$. After washing in $1 \mathrm{~mol} / \mathrm{L}$ phosphate-buffer saline (PBS, $\mathrm{pH} 7.4$ ), the sections were covered with normal serum in a humidity chamber for $30 \mathrm{~min}$ at room temperature. Excess serum was rinsed off with $1 \mathrm{~mol} / \mathrm{L} \mathrm{PBS}$, and the sections were incubated with the primary antibodies in a humidity chamber for $45 \mathrm{~min}$ at room temperature. Then sections were rinsed with PBS before incubation with the biotinylated second antibody in a humidity chamber for $40 \mathrm{~min}$ at $37^{\circ} \mathrm{C}$. After rinsing with $\mathrm{PBS}$, the streptavidin-peroxidase complex reagent (StrepABComplex/HRP DUET, CAKO) was added. Slides were incubated for $45 \mathrm{~min}$ at room temperature, then washed in $1 \mathrm{~mol} / \mathrm{L}$ PBS, and covered with 3,3'-diaminobenzidine tetrahydrochloride solution for 15 min under the microscope. Sections were then immersed in running tap water, counterstained with hematoxylin for $1 \mathrm{~min}$, followed by a tap water bath, immersion in a series of alcohol baths of increasing concentrations and xylene, and then covered with coverslips. Negative controls were performed in which the primary antibody was omitted. The slides were performed using a mouse monoclonal anti-OPN antibody (abcam) in the same way as the slides for OPN staining.

\section{Double-labeling immunohistochemistry}

OPN and CEACAM5 were performed by double staining against OPN (abcam) and CEACAM5 (abcam) according to the manufacturer's instructions (Zymed HISTOSTAIN-DS BROAD SPECTRUM). Each antibody labeling was performed as described above. Tween-20 PBS (0.05\% TPBS, pH 7.4) was used as the buffer. Staining was performed consecutively with CEACAM5 as the first staining. CEACAM5 and OPN labelings were performed by using different chromogens (AEC AND $\mathrm{BCIP} / \mathrm{NBT}$, respectively). Negative controls were performed by displacing primary antibodies with PBS.

\section{Evaluation of CEACAM5 and OPN staining}

Histological and IHC evaluations were independently made by three pathologists. Slides with debating evaluation were re-evaluated, and a consensus was reached. For each sample, at least 3000 carcinoma cells were evaluated for CEACAM5 or OPN expression. We examined the sections at $200 \times$ magnification, and the percentage of carcinoma cells with membranous or cytoplasmic staining was determined.

\section{Statistical analysis}

Statistical annlysis was performed by using SPSS 10.0 software package for Windows. The chiaquare test was used to analyze the data. Differences were considered significant at $\mathrm{P}<0.05$.

\section{Result}

\section{OPN, CEACAM5 expression and colocalization}

Our IHC study showed that OPN and CEACAM5 expression were found in normal tongue tissue, but with different expression pattern and location. CEACAM5 expression with membranous staining was restricted on the superficial epithelium (mainly granular layer, Figure 1A). However, OPN expression with mainly cytoplasmic staining was restricted on the deep epithelium (mainly basal layer, Figure 1B). Fourteen out of all 40 normal tissue CEACAM5 was expressed with membranous staining, six cases with a cytoplasmic staining and five with a mixed pattern of cytoplasmic and membranous staining. Twenty out of all 40 normal tissue expressed OPN with cytoplasmic staining, six cases expressed OPN with membranous staining, seven cases expressed OPN with a mixed pattern of cytoplasmic and membranous staining. The expression patterns showed a significant different between OPN and CEACOM5 in normal tissue (Table 1). According to dual-labeling IHC 


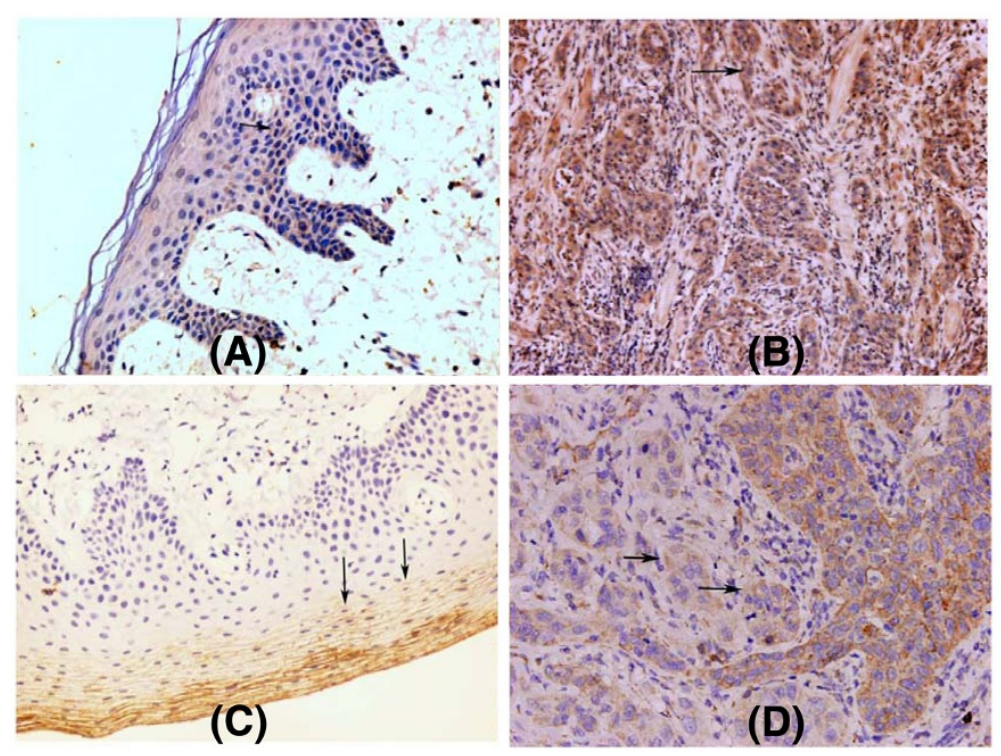

Figure 1 In both of normal tissue and carcinoma, OPN were all expressed with mainly cytoplasmic staining (arrow) (A and B).

CEACAM5 expression was found in normal tongue tissue and carcinoma with different expression pattern and location. CEACAM5 expression with membranous staining was restricted on the superficial epithelium(arrow) (C) in normal tissue. However, CEACAM5 expression with mainly cytoplasmic staining was restricted on the deep epithelium (arrow) (D) in squamous carcinoma of tongue.

staining of CEACOM5 and OPN, both of them were expressed in the normal tissue, and no colocalization was observed in cytoplasm (Figure 2A).

In squamous carcinoma of tongue, 59 of 80 analyzed tumor samples have been found to express OPN with

Table 1 Clinicopathological Characteristics of the Patients

\begin{tabular}{lllll} 
Postive samples & & \multicolumn{2}{c}{ Staining pattern } & p values \\
&
\end{tabular}

\section{CEACAM5}

Normal

Carcinoma

25

68

$\begin{array}{ccc}14 & 6 & 5 \\ 6 & 56 & 6\end{array}$

OPN

Normal

Carcinoma

33

70

$\begin{array}{lll}6 & 20 & 7 \\ 6 & 59 & 5\end{array}$

Normal tissue

OPN

CEACAM5

25
33

$\begin{array}{ccc}14 & 6 & 5 \\ 6 & 20 & 7\end{array}$

Carcinoma

$\begin{array}{lllll}\text { OPN } & 70 & 6 & 56 & 5 \\ \text { CEACAM5 } & 68 & 6 & 56 & 6\end{array}$

M: membranous; C: cytoblasmic; $\mathbf{M}+\mathbf{C}:$ membranous and cytoblsmic. Significant, $P<0.05$. cytoplasmic staining, six cases with membranous staining and five cases with a mixed pattern of cytoplasmic and membranous staining. 56 of 80 analyzed tumor samples expressed CEACOM5 with cytoplasmic staining, six cases with membranous staining and six cases with a mixed pattern of cytoplasmic and membranous staining in all of 68 analyzed positive tumor samples (Figure 1C and D). CEACAM5 expression with cytoplasmic staining was different from normal tongue tissue with membranous staining. The expression patterns of CEACOM5 showed a significant difference between normal tissue and the squamous carcinoma (Table 1). In addition, we could demonstrate that 42 in all of 80 tumor samples, CEACAM5 and OPN are being colocalized in cytoplasm (Figure 2B) by using double-labeling immunohistochemistry. A significant correlation was observed between the positive colocalization and the negative colocalization in the depth of tumor invasion and differentiation (Table 2). Dual-labeling IHC showed that the movement of CEACAM5 from membrane to cytoplasm may promote OPN expression, and more colocalization cases were detected in squamous carcinoma of tongue. The findings demonstrated that the transformation of CEACAM5 expression pattern and colocalization with OPN might play a critical role in tumor metastasis and invasion.

\section{Discussion}

The human CEACAM5 protein family encompasses several forms of proteins with different biochemical features. CEACAM5 is an oncofetal glycoprotein, 


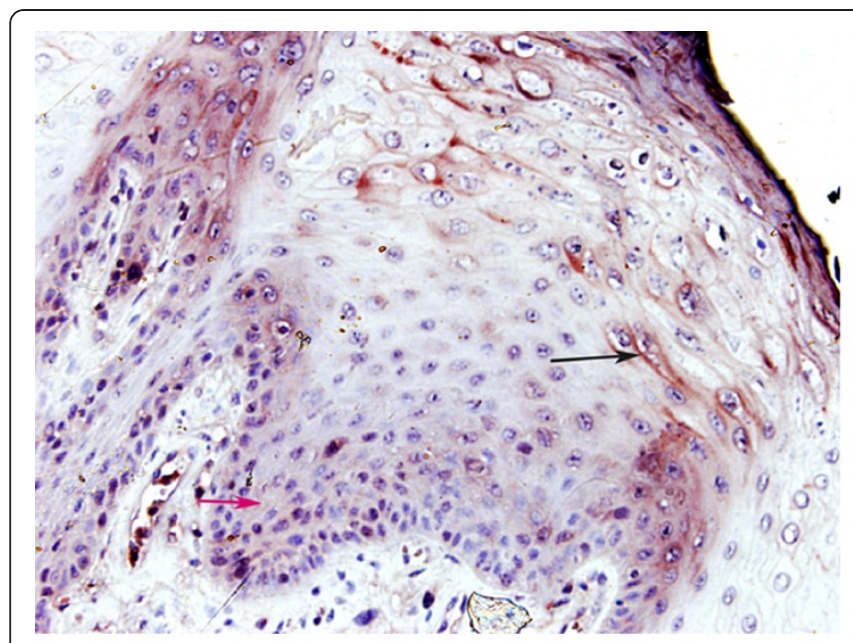

(A)

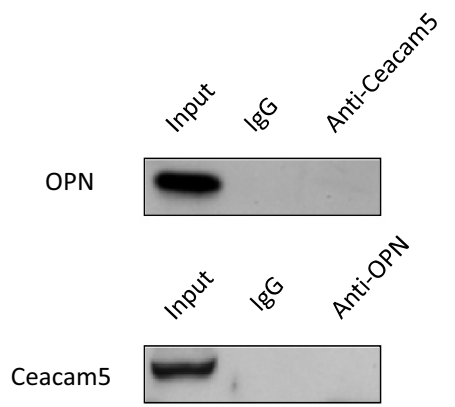

(C)

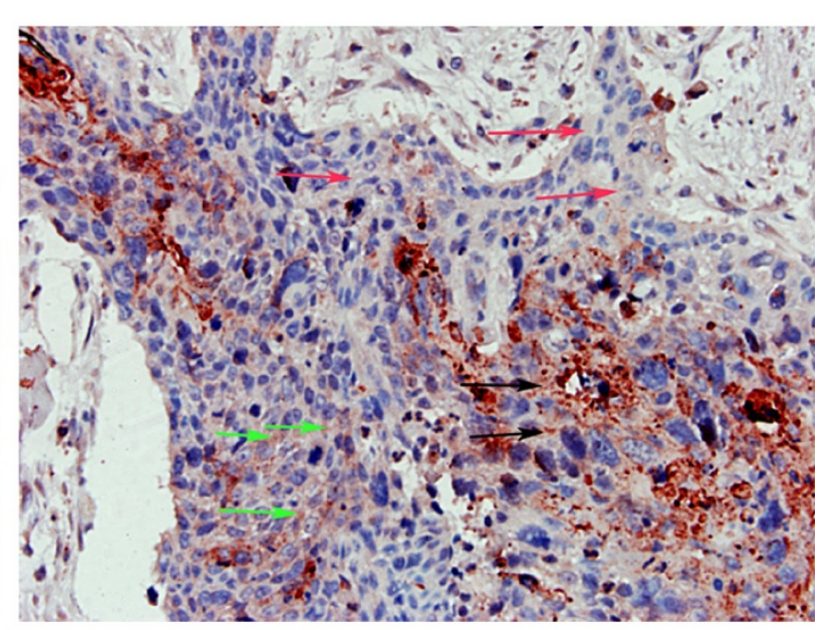

(B)

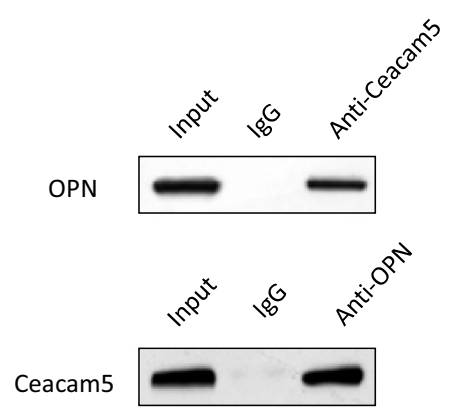

(D)

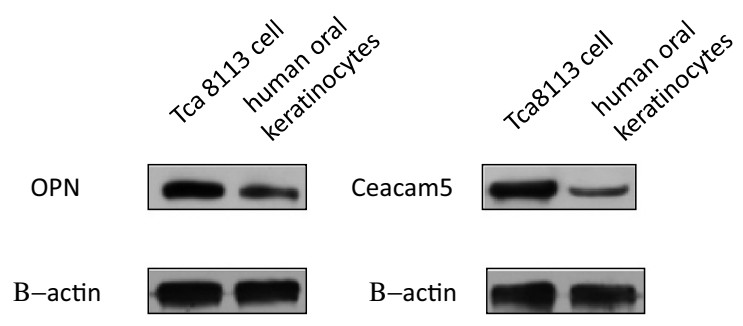

(E)

Figure 2 OPN and CEACAM5 expression with different expression pattern and location were found in normal tongue tissue. CEACAM5 expression with membranous staining was restricted on the superficial epithelium (black arrow) (A), however, OPN expression with mainly cytoplasmic staining was restricted on the deep epithelium (red arrow) (A). no colocalization was observation in cytoplasm (A). In squamous carcinoma of tongue, OPN (red arrow) and CEACAM5 (black arrow) expression were found with cytoplasmic staining (B). CEACAM5 and OPN are being colocalized in cytoplasm (green arrow) (B).

containing 50\% carbohydrate with a molecular weight of approximately $200 \mathrm{kDa}$ [17]. CEACAM5 is overexpressed in several tumor types of epithelial origin and is known as an important and extensively used clinical tumor marker for colorectal and other carcinomas [18]. Hence, CEACAM5 is an attractive target for immunotherapeutic purposes because of its expression profile, its role in tumor progression, and its immunogenicity. In benign and malignant lesions of human carcinoma of tongue, the apical membrane expression of CEACAM5 changes to a distinct uniform membrane staining in an early stage of malignant transformation, and the incident of the uniform membrane expression might be due to a loss of or reduction in the interaction among the adhesion molecules with its binding molecules, implicating an important shift towards the malignant phenotype [19]. Using immunohistochemistry, we found that normal tissue of tongue expressed CEACAM5 with apical 
Table 2 Clinicopathologica Characteristics of the Patients

\begin{tabular}{lcccc}
\hline Variables & No.of patients & \multicolumn{2}{c}{ Colocalization } & p \\
\cline { 3 - 4 } & & Negative & Positive & \\
\hline Tissue & 80 & 40 & 0 & \\
Normal & 40 & 42 & 38 & \\
Carcinoma & & & & \\
Carcinoma & & & & \\
Differentiation & 43 & 16 & 27 & \\
Undifferentiated & 37 & 28 & 9 & \\
Differentiated & & & & \\
Carcinoma & & & & \\
Depth of invasion & & 29 & 16 & \\
$T_{1-2}$ & 45 & 14 & 21 & \\
$T_{3-4}$ & 35 & & & $<0.05$ \\
& & & &
\end{tabular}

Significant, $\mathrm{P}<0.05$.

and uniform membranous patterns, and the carcinomas mainly expressed CEACAM5 with a cytoplasmic pattern. Therefore, membranous distribution of CEACAM5 changed to cytoplasmic staining may indicate a malignant transformation .

OPN is a calcium-binding phosphoprotein with multiple functions. Under physiological conditions, OPN is produced by osteoblasts when stimulated by calcitriol, and it functions by binding to hydroxyapatite to provide the anchoring of osteoclast to the mineral of bone matrix [20]. OPN binds to cells via the vitronectin receptor but also via other integrins and the hyaluronic acid receptor CD44. Several studies have defined OPN as an important glycoprotein with multiple functions and have found it plays a role in basic cellular processes, such as neovascularization and tissue remodeling, which are essential to metastasis and invasion of the tumor $[21,22]$. Furthermore, several lines of evidence have implicated OPN in angiogenesis, and vascular endothelilal growth factor may induce expression of OPN as well as $\alpha v$ B3 integrin in endothelial cells [23-25]. Overexpression of OPN in tongue cancers implicated a more aggressive tumor behavior and was an important factor for survival $[26,27]$. Having assessed the distribution of OPN protein by immunohistochemistry, we found that OPN is expressed in a large percentage of the tissue studied. Significant cytoplasmic OPN staining was observed in a large percentage of all tissue studied.

In the present study, we have investigated the expression pattern of OPN at all tissue studied and its correlation with the expression of CEACAM5. As shown by immunohistochemistry, significant cytoplasmic OPN staining was observed in a large percentage of all tissue studied. CEACAM5 expression with membranous staining was found in normal tongue tissue. In squamous carcinoma of tongue, CEACAM5 expression with cytoplasmic staining was different from normal tongue tissue with membranous staining. According to dual-labeling IHC staining of CEACOM5 and OPN, both of them were expressed in the normal tissue, and no colocalization was observed. However, CEACAM5 and OPN are being colocalized in squamous carcinoma of tongue, and a significant correlation was observed between the positive colocalization and the negative colocalization in the depth of tumor invasion and the differentiation. OPN and CEACAM5 were expressed in both normal and pathological tongue tissues, and their different expression pattern can be potentially useful as an additional diagnostic marker in squamous carcinoma of tongue. Julianne Briese et al. have demonstrated that OPN is colocalized with the CEACAM1 in the extravillous trophoblast of the human placenta and enhances invasion of CEACAM1-expressing placental cells [16]. We have performed a systematic immumohistochemical study on OPN and CEACAM5 protein expression in squamous carcinoma of tongue. Limitations of our study include the absence of in vitro experiment and functional studies. Nevertheless, with the IHC of the correlated expression pattern of OPN and CEACAM5 in carcinoma, our data showed that OPN and CEACAM5 may act as a functional complex in these lesions. This complex may increase invasion and inhibit differentiation of the carcinoma of tongue.

\section{Competing interests}

The authors declare that they have no competing interests.

\section{Authors' contributions}

FZ and FCW designed the project. All authors performed the experiment. FZ drafted the manuscript. All authors read and approved the final manuscript.

\section{Author details}

'Burns and Plastic Surgery of Qilu hospital of Shandong University, 107\#, Wenhua Xi Road, Jinan, Shandong 250012, People's Republic of China. ${ }^{2}$ Hepatology Department of Qilu hospital of Shandong University, 107\#, Wenhua Xi Road, Jinan, Shandong 250012, People's Republic of China. ${ }^{3}$ Institute of Basic Medical Sciences of Qilu hospital of Shandong University, 107\#, Wenhua Xi Road, Jinan, Shandong 250012, People's Republic of China. ${ }^{4}$ Qilu hospital of Shandong University, 107\#, Wenhua Xi Road, Jinan, Shandong 250012, People's Republic of China. ${ }^{5}$ Oral and Maxillofacial Surgery of Qilu hospital of Shandong University, 107\#, Wenhua Xi Road, Jinan, Shandong 250012, People's Republic of China.

Received: 16 March 2012 Accepted: 4 June 2012

Published: 27 June 2012

\section{References}

1. Steller H: Mechanisms and genes of cellular suicide. Science 1995, 267:1445-1449.

2. Thompson CB: Apoptosis in the pathogenesis and treatment of disease. Science 1995, 267:1456-1462.

3. Liaw L, Almeida M, Hart CE, Schwarz SM, Giachelli CM: The adhesive and migratory effects of osteopontin are mediated via distinct cell surface 
integrins: role of alpha $v$ beta 3 in smoothmuscle cellmigration to osteopontin in vitro. J Clin Invest 1995, 95:713-724.

4. Daiter E, Omigbodun A, Wang S, Walinsky D, Strauss JF III, Hoyer JR, Coutifaris C: Cell differentiation and endogenous cyclic adenosine 3,5,monophosphate regulate osteopontin expression in human trophoblasts. Endocrinology 1996, 137:1785-1790.

5. Yue TL, McKenna PJ, Ohlstein EH, Farach-Carson MC, Butler WT, Johanson K, McDevitt P, Feuerstein GZ, Stadel JM: Osteopontin-stimulated vascular smooth muscle cell migration is mediated by beta 3 integrin. Exp Cell Res 1994, 214:459-464.

6. Johnson GA, Burghardt RC, Bazer FW, Spencer TE: Osteopontin: roles in implantation and placentation. Biol Reprod 2003, 69:1458-1471.

7. Brown LF, Berse B, Van de Water L, Papadopoulos-Sergiou A, Perruzzi CA, Manseau EJ, Dvorak HF, Senger DR: Expression and distribution of osteopontin in human tissues. Biol Cell 1992, 3:1169-1180.

8. Rittling SR, Novick KE: Osteopontin expression in mammary gland development and tumorigenesis. Cell Growth Differ 1997, 8:1061-1069.

9. Beauchemin N, Draber P, Dveksler G, Gold P, Gray-Owen S, Grunert F, Hammerstrom S, Holmes KV, Karlsson A, Kuroki M, Lin SH, Lucka L, Najjar SM, Neumaier M, Obrink B, Shively JE, Skubitz KM, Stanners CP, Thomas $P$, Thompason JA, Virji M, von Kleist S, Wagener C, Watt S, Zimmermann W: Redefined nomenclature for members of the carcinoembryonic antigen family. Exp Cell Res 1999, 252:243-249.

10. Gold P, Freedman SO: Specific carcinoembryonic antigens of the human digestive system. J Exp Med 1965, 122:467-481.

11. Goldenberg DM, Sharkey RM, Primus FJ: Carcinoembryonic antigen in histopathology: immunoperoxidase staining of conventional tissue sections. J Natl Cancer Inst 1976, 57:11-22.

12. Shively JE, Beatty JD: CEA-related antigens: molecular biology and clinical significance. Crit Rev Oncol Hematol 1985, 2:355-399.

13. Thompson JA, Grunert F, Zimmermann W: Carcinoembryonic antigen gene family: molecular biology and clinical perspectives. J Clin Lab Anal 1991, 5:344-366.

14. Gold P, Goldenberg NA: The carcinoembryonic antigen (CEA): past present, and future. McGill J Med 1997, 3:46-66.

15. Hammarstrom S: The carcinoembryonic antigen (CEA) family: structures, suggested functions and expression in normal and malignant tissues. Semin Cancer Biol 1999, 9:67-81.

16. Briese J, Oberndorfer M, Patschenik C, Schulte HM, Makriqiannakis A, Loning T, Bamberger AM: Osteopontin Is Colocalized with the Adhesion Molecule CEACAM1 in the Extravillous Trophoblast of the Human Placenta and Enhances Invasion of CEACAM1-Expressing Placental Cells. J Clin Endocrinol Metab 2005, 90:5407-5413.

17. Obrink B: CEAadhesion molecules: multifunctional proteins with signalregulatory properties. Curr Opin Cell Bior 1997, 9:616-626.

18. Beauchemin N, Kunath T, Tobitaille J, et al: Association of biliary glycoprotein with protein tyrosine phosphatase SHP-1 in malignant colon epithelial cells. Oncogene 1997, 14:783-790.

19. Lutz Riethdorf L, Lisboa BW, Henkel U, Naumann M, Wagener C, Loning T: Differential expression of CD66a (BGP), a cell adhesion molecule of the carcinoembryonic antigen family, in benign, premalignant, and malignant lesions of the human mammary gland. J Histochem Cytochem 1997, 45:957-964.

20. Giachelli CM, Steitz S: Osteopontin: a versatile regulator of inflammation and biomineralization. Matrix Biol 2000, 19:615-622.

21. Senger DR, Ledbetter SR, Claffey KP, PapadopoulosSergiou A, Perruzzi CA, Detmar M: Stimulation of endothelial cell migration by vascular permeability factor/vascular endothelial growth factor through cooperative mechanisms involving the av $B 3$ integrin, osteopontin, and thrombin. Am J Pathol 1996, 149:293-305.

22. Denhardt DT, Guo X: Osteopontin: a protein with diverse functions. FASEB J 1993, 7:1475-1482.

23. Shijubo N, Uede T, Kon S, Nagata M, Abe S: Vascular endothelial growth factor and osteopontin in tumor biology. Crit Rev Oncog 2000, 11:135-146.

24. Morimoto J, Inobe M, Kimura C, Kon S, Diao H, Aoki M, Miyazaki T, Denhardt DT, Rittling S, Uede T: Osteopontin affects the persistence of $\beta$-glucaninduced hepatic granuloma formation and tissue injury through two distinct mechanisms. Int Immunol 2004, 16:477-488.

25. Coppola D, Szabo M, Boulware D, Muraca P, Alsarraj M, Chambers AF, Yeatman TJ: Correlation of osteopontin protein expression and pathological stage across a wide variety of tumor histologies. Clin Cancer Res 2004, 10:184-190.

26. Chien CY, Su CY, Fang FM, Huang HY, Chen $\mathrm{CM}$, Chen $\mathrm{CH}$, Huang CC: Clinical significance of osteopontin expression in $\mathrm{T} 1$ and $\mathrm{T} 2$ tongue cancers. Head Neck 2008, 30:776-781.

27. Chiu YW, Tu HF, Wang IK, Wu CH, Chang KW, Liu TY, Kao SY: The implication of osteopontin (OPN) expression and genetic polymorphisms of OPN promoter in oral carcinogenesis. Oral Oncol 2010, 46:302-306. Epub 2010 Mar 9.

doi:10.1186/1475-2867-12-33

Cite this article as: Zhang et al:: Osteopontin's colocalization with the adhesion molecule CEACAM5 in cytoplasm of carcinoma of tongue and its correlation with the invasion of that diease. Cancer Cell International 2012 12:33

\section{Submit your next manuscript to BioMed Central and take full advantage of:}

- Convenient online submission

- Thorough peer review

- No space constraints or color figure charges

- Immediate publication on acceptance

- Inclusion in PubMed, CAS, Scopus and Google Scholar

- Research which is freely available for redistribution 